\title{
Cilindros ocos 1-D com geração de calor: uma aproximação suficientemente geral de solução para problemas com condição de Dirichlet variável no tempo
}

\author{
Holow 1-D cylinders with heat generation: a sufficiently general approach to solving \\ problems with a time variable Dirichlet condition
}

\begin{abstract}
Jorge Corrêa de Araújo Universidade do Estado do Rio de Janeiro (UERJ), Faculdade de Formação de Professores (FFP), Departamento de Matemática (DMAT), São Gonçalo, RJ, Brasil https://orcid.org/0000-0002-1015-6311, jcaraujo_55@yahoo.com.br
\end{abstract}

\begin{abstract}
Rosa María García Márquez Universidade do Estado do Rio de Janeiro (UERJ), Faculdade de Formação de Professores (FFP), Departamento de Matemática (DMAT), São Gonçalo, RJ, Brasil https://orcid.org/0000-0003-3465-569X, rosagmarquez@yahoo.com.br
\end{abstract}

\author{
Informações do Artigo \\ Como citar este artigo \\ ARAÚJO, Jorge Corrêa de; MÁRQUEZ, Rosa \\ María García. Cilindros ocos 1-D com \\ geração de calor: uma aproximação \\ suficientemente geral de solução para \\ problemas com condição de Dirichlet variável \\ no tempo. REMAT: Revista Eletrônica da \\ Matemática, Bento Gonçalves, RS, v. 7, n. 1, \\ p. e3015, 30 jun. 2021. DOI: \\ https://doi.org/10.35819/remat2021v7i1id4638
}

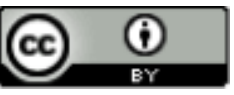

Histórico do Artigo

Submissão: 19 de outubro de 2020.

Aceite: 28 de janeiro de 2021.

\section{Resumo}

Neste trabalho, uma solução aproximada suficientemente geral para problemas de condução de calor transiente em geometria cilíndrica 1-D, com geração de calor e condições de Dirichlet variável no tempo foi apresentada usando o método de funções de Green. Uma importante integral envolvendo funções de Bessel, que faz parte da solução, foi aqui resolvida com detalhes. Os resultados obtidos com o uso dessa solução, quando aplicada em alguns casos particulares de interesse prático, ficaram em boa concordância com as soluções reportadas na literatura. Foi adotada uma metodologia que consiste em fatiar a solução do problema não homogêneo com condições de fronteira não homogêneas em uma solução do problema não homogêneo com condições de fronteira homogêneas mais duas soluções estacionárias relacionadas com as condições de Dirichlet dadas. Com isso, a solução obtida não tem problemas de convergência nas fronteiras da região cilíndrica com as condições prescritas de temperaturas.

Palavras-chave

Cilindro Oco

Funções de Bessel

Método de Funções de Green

Solução Geral Aproximada

Keywords

Hollow Cylinder

Bessel Functions

Green's Function Method

Approximated General Solution

\begin{abstract}
In this work, a sufficiently approximated general solution to transient heat conduction problems in 1-D cylindrical geometry, with heat generation and time variable Dirichlet conditions, was presented using the Green's function method. An important integral involving Bessel functions, that is part of the solution, has been solved in detail here. The results obtained from the use of this solution, when applied in some particular cases of practical interest, were aligned with the solutions reported by the literature. We have adopted, in this sense, a methodology that consists of addressing a non-homogeneous problem solution with non-homogeneous boundary conditions in a nonhomogeneous problem solution with homogeneous border conditions and two more stationary solutions related to the given Dirichlet conditions. As a result, the solution obtained has no problems of convergence at the boundaries of the cylindrical region with the temperature prescribed conditions.
\end{abstract}




\section{Introdução}

O problema de condução de calor em regime transiente em um longo e fino cilindro oco 1$\mathrm{D}$ de material homogêneo e isotrópico, com geração de calor $g(r, t)$ por unidade de tempo, por unidade de volume $\left(\frac{W}{\mathrm{~m}^{3}}\right)$ e com condições de Dirichlet variáveis, que segundo Hahn e Özisik (2012, p. 329) é dado matematicamente por

$$
\frac{\partial^{2} T}{\partial r^{2}}+\frac{1}{r} \frac{\partial T}{\partial r}+\frac{g(r, t)}{k}=\frac{1}{\kappa} \frac{\partial T}{\partial t}, \quad a<r<b, \quad t>0,
$$

onde $T=T(r, t)$ é a temperatura na região cilíndrica, $k$ é a condutividade térmica do material, enquanto $\kappa$ é a difusividade térmica da substância, isto é, representa as propriedades térmicofísicas do meio (HAHN; ÖZISIK, 2012). Junto à equação (1) são impostas condições de fronteira do tipo Dirichlet, $T_{S}=f(t)$ e uma condição inicial dada que são representadas pelas equações:

$$
\begin{aligned}
& \left\{\begin{array}{l}
C F 1: \quad T(a, t)=f_{1}(t), \quad t>0, \\
C F 2: \quad T(b, t)=f_{2}(t), \quad t>0,
\end{array}\right. \\
& C I: \quad T(r, 0)=F(r), \quad a \leq r \leq b .
\end{aligned}
$$

Os cilindros ocos têm grande importância prática, em especial na engenharia. Por exemplo, na mecânica, os cilindros de pistão oco hidráulicos são largamente utilizados. Na eletricidade, esses cilindros são feitos de discos de ferrite, que é um material formado por óxido de ferro com propriedades magnéticas, são usados comumente para evitar variações elevadas de temperaturas e picos de calor nas correntes elétricas que passam por cabos, como o fio de um notebook (ROMER, 2013). Esses cilindros são também usados como encamisantes em camadas cilíndricas onde há geração de calor, como, por exemplo, em usinas nucleares e termoelétricas. Segundo Rodrigues e Mesquita (2017), elementos combustíveis de hidreto de urânio-zircônio de diâmetro $D=37,7 \mathrm{~mm}$ encapsulados em alumínio tipo AL110F de espessura $e=0,76 \mathrm{~mm}$ são utilizados no reator de pesquisa Triga IPR-R1, localizado no Campus da UFMG. Essas ligas de alumínio são adotadas em larga escala como encamisantes em reatores de elemento combustível a base de urânio $(\mathrm{U})$, plutônio $(\mathrm{Pu})$ ou tório $(\mathrm{Th})$ devido à sua elevada condutividade térmica, baixa seção de captura de nêutrons térmicos, boa resistência à corrosão, disponibilidade e baixo custo (PERROTTA, 1999). Sabemos que, em geral, uma análise unidimensional não é realista para um problema 3-D, e mesmo movimentos bidimensionais de um fluido, dependentes do tempo, não existem na realidade (MEYER, 2007). Entretanto, Fox, Pritchard e McDonald (2015) afirmam que em muitos problemas na engenharia, mesmo uma análise 1-D é adequada para fornecer soluções aproximadas dentro da precisão desejada. Nesse sentido, os cilindros circulares infinitos, isto é, longos, $\frac{L}{r_{0}} \geq 10$, e finos, são adequados a uma análise unidimensional por atenderem ao requisito 
da simetria radial, $\partial_{r} T(0, t)=0$. Desse modo, muitos problemas de importância prática podem ser aproximados pela formulação matemática descrita pelas equações (1) a (3). Se as condições de fronteira são funções do tempo, o método de separação de variáveis deve ser descartado (FERNANDES, 2009). Embora a transformada de Laplace tenha sido muito utilizada por Carslaw e Jaeger (2011) para a obtenção de uma solução geral para problemas de condução de calor homogêneo, transiente em cilindros ocos 1-D, ela não contempla condições de Dirichlet mais gerais do que valores constantes, e, além disso, é necessário que a temperatura inicial sobre a região cilíndrica seja zero.

Cinelli (1965) apresentou um artigo no qual usou o método da transformada Hankel finita para resolver problemas de condução de calor nessa geometria, de modo a estudar com mais ênfase as condições de fronteira de Cauchy, $\partial_{S} T=h\left(T_{S}-T_{\infty}\right)$ sobre as faces da região, que ocorre, por exemplo, em reatores nucleares. As condições de Cauchy são mais fáceis de realizar do ponto de vista físico, como, por exemplo, um sistema de ventilação que desloca um fluido, como o ar mais frio de um ponto para outro, enquanto uma condição de Dirichlet pode representar uma mudança de fase de uma substância que ocorre na superfície, como a evaporação/cozimento (HAHN; ÖZISIK, 2012). Segundo Hahn e Özisik (2012), o método mais poderoso para resolver problemas de condução de calor transiente e não homogêneo é de aproximação por funções de Green. A grande força do uso dessas funções está exatamente na possibilidade de obtenção de soluções, desse tipo, de problemas mais variados e complexos, incluindo não homogeneidades que variam no tempo e espaço (FERNANDES, 2009). Para obter a função de Green é preciso resolver o problema homogêneo associado por separação de variáveis. Uma vez obtida a função de Green para um dado problema específico, a solução analítica de distribuição de temperatura é imediatamente disponível. Essa solução envolve vários termos, e cada um deles tem um significado físico (HAHN; ÖZISIK, 2012). Entretanto, quando são usadas condições de Dirichlet temporais como as especificadas pelas equações (2) e (3), a solução obtida usando funções de Green pode não convergir uniformemente nas regiões próximas aos pontos de fronteira, como pode ser visto no exemplo 8.8 de Hahn e Özisik (2012, p. 329).

Com o objetivo de remover, ou mesmo atenuar essa dificuldade, Hahn e Özisik (2012) sugerem o fatiamento da solução original, e o problema não homogêneo com condições de fronteira não homogêneas é transformado em um problema não homogêneo, mas agora com condições de fronteira homogêneas. Nosso objetivo é usar o método de funções de Green para obter uma expressão suficientemente geral, que seja uma aproximação da solução para problemas de condução de calor em regime transiente, com geração de calor e com condições de fronteiras não homogêneas do tipo Dirichlet variando com o tempo, e uma dada condição inicial na geometria 1-D em cilindros ocos finos e longos, ou seja, problemas de calor que podem ser representados pelas equações (1) a (4). Uma importante integral envolvendo funções de Bessel que faz parte da aproximação da solução geral estabelecida pode ser resolvida, o que produziu 
simplificações nessa equação quando casos particulares dela foram analisados. Alguns desses casos especiais reportados na literatura existente foram utilizados para a validação por comparação da solução geral quando aplicada a cada caso analisado.

\section{Materiais e métodos}

A equação de condução de calor homogênea em regime transiente, e em um sistema 1-D em coordenadas cilíndricas, é dado pela equação (CARSLAW; JAEGER, 2011):

$$
\frac{\partial^{2} T}{\partial r^{2}}+\frac{1}{r} \frac{\partial T}{\partial r}=\frac{1}{\kappa} \frac{\partial T}{\partial t}
$$

\subsection{O problema de Sturm-Liouville em coordenadas cilíndricas}

Uma equação da forma

$$
\frac{d}{d x}\left[p(x) \frac{d y}{d x}\right]+[q(x)+\lambda w(x)] y=0
$$

que satisfaz certas condições de fronteira em um intervalo $a \leq x \leq b$, sendo $p(x), q(x)$ e $w(x)$ funções contínuas nesse intervalo, com $p(x)$ diferenciável, é chamada equação de SturmLiouville. A busca de soluções não triviais para essa equação com as condições de fronteira nos extremos do intervalo é chamada problema de Sturm-Liouville (DAVIS, 1963, SOTOMAYOR, 1979). Tais soluções são denominadas autofunções, enquanto os $\lambda$ 's correspondentes são denominados autovalores. Esse problema em coordenadas cilíndricas é dado como na equação (5) substituindo a variável $x$ por $r$, isto é,

$$
\frac{d}{d r}\left[p(r) \frac{d y}{d r}\right]+[q(r)+\lambda w(r)] y=0 .
$$

Em particular, se $p(r)=r, q(r)=-\frac{v}{r} \quad$ e $w(r)=r$, e fazendo $y=R$, tem-se a equação diferencial ordinária na variável $r$ dada por

$$
\frac{d^{2} R}{d r^{2}}+\frac{1}{r} \frac{d R}{d r}+\left(\lambda^{2}-\frac{v^{2}}{r^{2}}\right) R=0
$$

onde $r>0$ e geralmente $v=0,1,2 \ldots$.

A equação (7) sujeita às condições de fronteira homogêneas dadas por

$$
\begin{aligned}
& A_{1} \frac{d R}{d r}+A_{2} R=0, \quad r=a, \\
& B_{1} \frac{d R}{d r}+B_{2} R=0, \quad r=b,
\end{aligned}
$$

onde $A_{1}, A_{2}, B_{1}, B_{2}$ são constantes não nulas, é um problema de Sturm-Liouville em coordenadas cilíndricas (ou esféricas). As autofunções $R_{v}\left(r, \lambda_{n}\right)$, sob a condição de $\lambda>0$ (HAHN; 
ÖZISIK, 2012) que satisfazem a equação (7) e as condições dadas pela equação (8), constituem um conjunto ortogonal de funções no espaço $C^{2}([a, b])$ com respeito ao produto interno dado por

$$
\langle f, g\rangle=\int_{a}^{b} r f(r) g(r) d r
$$

sendo a norma quadrática de cada autofunção dada por (ÖZISIK; HAHN, 2012)

$$
N\left(\lambda_{n}\right)=N\left[R_{v}\left(r, \lambda_{n}\right)\right]=\int_{a}^{b} r R_{v}^{2}\left(r, \lambda_{n}\right) d r .
$$

Segundo Davis (1963), toda função $R$ integrável no espaço $C^{2}([a, b])$, que satisfaz as condições de fronteiras dadas pela equação (8), pode ser expandida em uma série que converge ponto a ponto neste intervalo, isto é,

$$
R(r)=\sum_{n=1}^{\infty} C_{n} R_{v}\left(r, \lambda_{n}\right)
$$

onde $C_{n}=\frac{1}{N\left(\lambda_{n}\right)} \int_{a}^{b} r R(r) R_{v}\left(r, \lambda_{n}\right) d r, \quad n=1,2, \ldots$

A equação (7) é chamada de equação de Bessel e tem como solução geral a equação dada por (HAHN; ÖZISIK, 2012)

$$
R(r)=c_{1} J_{v}(\lambda r)+c_{2} Y_{v}(\lambda r),
$$

onde $J_{v}(\lambda r)$ e $Y_{v}(\lambda r)$ são, respectivamente, as funções de Bessel de primeira e segunda espécie de ordem $v$.

\subsection{Método de Separação de Variáveis}

Um método padrão para obter uma solução da equação (4) é assumir que as variáveis são separáveis, isto é,

$$
T(r, t)=G(r) H(t),
$$

onde $G$ e $H$ são só funções de $r$ e $t$, respectivamente. A substituição da equação (13) na equação (4) resulta em duas equações diferenciais ordinárias (EDOs) independentes na forma (HAHN; ÖZISIK, 2012)

$$
\frac{d^{2} G}{d r^{2}}+\frac{1}{r} \frac{d G}{d r}=\frac{1}{\kappa H} \frac{d H}{d t}=-\lambda^{2},
$$

ou seja,

$$
\begin{gathered}
\frac{1}{\kappa H} \frac{d H}{d t}=-\lambda^{2}, \\
\frac{d^{2} G}{d r^{2}}+\frac{1}{r} \frac{d G}{d r}+\lambda^{2} G=0 .
\end{gathered}
$$


A equação (15) pode ser resolvida diretamente por separação de variáveis para obter

$$
H(t)=c_{1} e^{-\kappa \lambda^{2} t}
$$

enquanto a EDO na variável $r$ é uma equação de Bessel de ordem $v=0$, cuja solução geral é dada pela equação (12), na forma

$$
G(r)=C_{2} J_{0}(\lambda r)+C_{3} Y_{0}(\lambda r),
$$

onde as constantes poderão ser eliminadas por meio de condições iniciais adequadas. Portanto, uma solução geral da equação (4) é dada pelo produto das soluções individuais dadas pelas equações (17) e (18), isto é,

$$
T(r, t)=C_{1} e^{-\kappa \lambda^{2} t}\left[C_{2} J_{0}(\lambda r)+C_{3} Y_{0}(\lambda r)\right]
$$

ou, omitindo as constantes,

$$
T(r, t)=e^{-\kappa \lambda^{2} t} G_{0}(r, \lambda) .
$$

Como, para cada $\lambda_{n}>0$, podemos associar uma autofunção $G_{v}\left(r, \lambda_{n}\right)$ e, desde que a equação (4) seja linear, a solução mais geral será obtida pela soma de todos os produtos das equações (17) e (18)

$$
T(r, t)=\sum_{n=1}^{\infty} C_{n} e^{-\kappa \lambda_{n}^{2} t} G_{0}\left(r, \lambda_{n}\right)
$$

onde os coeficientes $C_{n}$ são obtidos da equação (11) quando a condição inicial $T(r, 0)=F(r)$ é conhecida.

\section{Resultados e discussões}

A versão homogênea do problema dado pelas equações (1) a (4) fica na forma

$$
\begin{gathered}
\frac{\partial^{2} \psi}{\partial r^{2}}+\frac{1}{r} \frac{\partial \psi}{\partial r}=\frac{1}{\kappa} \frac{\partial \psi}{\partial t}, \quad a<r<b, \quad t>0, \\
C F 1: \quad \psi(a, t)=0, \quad t>0, \\
C F 2: \quad \psi(b, t)=0, \quad t>0, \\
C I: \quad \psi(r, 0)=F(r), \quad a \leq r \leq b .
\end{gathered}
$$

Usando o método de separação de variáveis, descrito na seção 2.2, tem-se das equações (13) e (18) e da condição $C F 2$, dada pela equação (24), que

$$
C_{3}=-C_{2} \frac{J_{0}(\lambda b)}{Y_{0}(\lambda b)},
$$

onde $Y_{0}(\lambda b) \neq 0$, pois, caso contrário, só teríamos a solução trivial. Das equações (18) e (26), resulta 


$$
G(r)=C_{4}\left[J_{0}(\lambda r) Y_{0}(\lambda b)-J_{0}(\lambda b) Y_{0}(\lambda r)\right]=C_{4} G_{0}(\lambda r)
$$

com $C_{4}=\frac{C_{2}}{Y_{0}(\lambda b)}$. Das equações (13) e (27) e da condição $C F 1$, dada pela equação (23), tem-se a equação transcendental

$$
G_{0}(\lambda a)=0
$$

onde

$$
G_{0}(\lambda r)=\left[J_{0}(\lambda r) Y_{0}(\lambda b)-J_{0}(\lambda b) Y_{0}(\lambda r)\right]
$$

é uma autofunção associada ao autovalor $\lambda$, definida no intervalo $a \leq r \leq b$.

As raízes $\lambda_{n}, \quad n=1,2, \ldots$ são todas reais e simples, onde para cada raiz $\lambda$ existe uma corresponde raiz $-\lambda$ (CARSLAW; JAEGER, 2011). Das equações (13), (17) e (27), tem-se

$$
\psi(r, t)=\sum_{n=1}^{\infty} C_{n} G_{0}\left(\lambda_{n} r\right) e^{-\kappa \lambda_{n}^{2} t}, \quad a<r<b, \quad t>0,
$$

de modo que os coeficientes $C_{n}$ são obtidos como na equação (11), trocando $R(r)$ por $F(r)$, isto é,

$$
C_{n}=\frac{1}{N\left(\lambda_{n}\right)} \int_{a}^{b} r G_{0}\left(\lambda_{n} r\right) F(r) d r
$$

sendo o inverso da norma dada, usando a equação (10),

$$
\frac{1}{N\left(\lambda_{n}\right)}=\frac{1}{\int_{a}^{b} r G_{0}^{2}\left(\lambda_{n} r\right) d r}
$$

Caso tivéssemos na equação (26) usado a condição $C F 1$, dada pela equação (23), em vez da $C F 2$, como foi realizado, poderíamos usar o caso 4, da Tabela 2.3 de Hahn e Özisik (2012, p. 54), para obter $\frac{1}{N\left(\lambda_{n}\right)}$ diretamente. Das equações (30) e (31), tem-se

$$
\psi(r, t)=\sum_{n=1}^{\infty} \frac{G_{0}\left(\lambda_{n} r\right)}{N\left(\lambda_{n}\right)} e^{-\kappa \lambda_{n}^{2} t} \int_{a}^{b} G_{0}\left(\lambda_{n} r^{\prime}\right) F\left(r^{\prime}\right) r^{\prime} d r^{\prime}=\int_{a}^{b} \sum_{n=1}^{\infty} \frac{G_{0}\left(\lambda_{n} r\right)}{N\left(\lambda_{n}\right)} e^{-\kappa \lambda_{n}^{2} t} G_{0}\left(\lambda_{n} r^{\prime}\right) F\left(r^{\prime}\right) r^{\prime} d r^{\prime} .
$$

De acordo com as equações (8) a (14) de Hahn e Özisik (2012, p. 306), a solução do problema homogêneo dado pelas equações (22) a (25) em termos de funções de Green pode ser colocada na forma

$$
\psi(r, t)=\int_{a}^{b} G\left(r, t, r^{\prime}, \tau\right)_{\tau=0} F\left(r^{\prime}\right) r^{\prime} d r^{\prime} .
$$

Portanto, por comparação das equações (33) e (34), pode-se obter a função de Green desenvolvida em $\tau=0$ para este problema, na forma 


$$
G\left(r, t, r^{\prime}, \tau\right)_{\tau=0}=\sum_{n=1}^{\infty} \frac{1}{N\left(\lambda_{n}\right)} G_{0}\left(\lambda_{n} r\right) e^{-\kappa \lambda_{n}^{2} t} G_{0}\left(\lambda_{n} r^{\prime}\right)
$$

ou, de modo geral,

$$
G\left(r, t, r^{\prime}, \tau\right)=\sum_{n=1}^{\infty} \frac{1}{N\left(\lambda_{n}\right)} G_{0}\left(\lambda_{n} r\right) e^{-\kappa \lambda_{n}^{2}(t-\tau)} G_{0}\left(\lambda_{n} r^{\prime}\right)
$$

que é a função de Green para a versão não homogênea dada pelas equações (22) a (25).

Em vez do uso de tabelas para a obtenção de $\frac{1}{N\left(\lambda_{n}\right)}$, Carslaw e Jaeger (2011) provaram de modo suscinto que

$$
N(\lambda)=\int_{a}^{b} r G_{0}^{2}(\lambda r) d r=\left.\frac{1}{2 \lambda^{2}}\left(r \frac{d G_{0}}{d r}\right)^{2}\right|_{a} ^{b}
$$

A equação (37) corresponde à equação (5) original dos referidos autores, onde $U_{0}(\alpha r)$ foi aqui substituído por $G_{0}(\lambda r)$. De acordo com as equações (7) e (8) de Carslaw e Jaeger (2011, p. 206), tem-se

$$
\left(r \frac{d G_{0}(\lambda r)}{d r}\right)_{r=a}=-\frac{2}{\pi \rho} \text { e }\left(\frac{r d G_{0}(\lambda r)}{d r}\right)_{r=b}=-\frac{2}{\pi}
$$

onde

$$
\rho=\frac{J_{0}(\lambda a)}{J_{0}(\lambda b)}
$$

Das equações (37) a (39), tem-se

$$
N\left(\lambda_{n}\right)=2 \frac{J_{0}^{2}(\lambda a)-J_{0}^{2}(\lambda b)}{\pi^{2} \lambda^{2} J_{0}^{2}(\lambda a)} .
$$

Das equaçõess (36) e (40), podemos expressar a função de Green como

$$
G\left(r, t, r^{\prime}, \tau\right)=\frac{\pi^{2}}{2} \sum_{n=1}^{\infty} \frac{\lambda_{n}^{2} J_{0}^{2}\left(\lambda_{n} a\right)}{J_{0}^{2}\left(\lambda_{n} a\right)-J_{0}^{2}\left(\lambda_{n} b\right)} e^{-\kappa \lambda_{n}^{2}(t-\tau)} G_{0}\left(\lambda_{n} r\right) G_{0}\left(\lambda_{n} r^{\prime}\right) .
$$

Em particular, quando a condição inicial for constante, a integral que aparece no lado direito da equação (33) pode ser simplificada. Carslaw e Jaeger (2011) apresentaram a seguinte proposição

$$
\int_{a}^{b} r G_{0}(\lambda r) d r=-\left.\frac{1}{\lambda^{2}}\left(r \frac{d G_{0}(\lambda r)}{d r}\right)\right|_{\grave{a}} ^{b}=\frac{2\left(J_{0}(\lambda a)-J_{0}(\lambda b)\right)}{\pi \lambda^{2} J_{0}(\lambda a)} .
$$

Aqui, o sinal negativo no termo do meio desta equação deveria ser positivo, pois, como está o lado direito da equação (42), fica com sinal oposto. Com o objetivo de dirimir este engano, vamos provar que 


$$
\int_{a}^{b} r G_{0}(\lambda r) d r=+\frac{1}{2 \lambda^{2}}\left[\left(\frac{r d G_{0}(\lambda r)}{d r}\right)\right]_{a}^{b}=\frac{2\left(J_{0}(\lambda a)-J_{0}(\lambda b)\right)}{\pi \lambda^{2} J_{0}(\lambda a)} .
$$

Da equação (29),

$$
\begin{aligned}
\int_{a}^{b} r G_{0}(\lambda r) d r & =Y_{0}(\lambda b) \int_{a}^{b} r J_{0}(\lambda r) d r-J_{0}(\lambda b) \int_{a}^{b} r Y_{0}(\lambda r) d r \\
& =Y_{0}(\lambda b)\left(\left.\frac{1}{\lambda} r J_{1}(\lambda r)\right|_{a} ^{b}\right)-J_{0}(\lambda b)\left(\left.\frac{1}{\lambda} r Y_{1}(\lambda r)\right|_{a} ^{b}\right),
\end{aligned}
$$

onde as expressões entre parênteses são obtidas da equação (22) de Hahn e Özisik (2012, p. 694). A equação (44) pode então ser colocada na forma

$$
\begin{aligned}
\int_{a}^{b} r G_{0}(\lambda r) d r= & \frac{b}{\lambda}\left\{Y_{0}(\lambda b)\left[\frac{d J_{0}(\lambda b)}{d r}\right]-J_{0}(\lambda b)\left[\frac{d Y_{0}(\lambda b)}{d r}\right]\right\}+ \\
& +\frac{a}{\lambda}\left\{J_{0}(\lambda b)\left[\frac{d Y_{0}(\lambda a)}{d r}\right]-Y_{0}(\lambda b)\left[\frac{d J_{0}(\lambda a)}{d r}\right]\right\} .
\end{aligned}
$$

Usando a equação (38) com os sinais corrigidos para mais, tem-se das equações (29) e (45) que

$$
\int_{a}^{b} r G_{0}(\lambda r) d r=\frac{b}{\lambda}\left[-\frac{1}{b \lambda}\right]\left[r \frac{d G_{0}(\lambda r)}{d r}\right]_{r=b}+\frac{a}{\lambda}\left[\frac{1}{a \lambda}\right]\left[r \frac{d G_{0}(\lambda r)}{d r}\right]_{r=a}=\frac{2\left(J_{0}(\lambda a)-J_{0}(\lambda b)\right)}{\pi \lambda^{2} J_{0}(\lambda a)} .
$$

A solução do problema de condução de calor dado pelas equações (1) a (4), em termos da função de Green dada pela equação (41), pode ser obtida usando as equações (8) a (14) de Hahn e Özisik (2012, p. 306), na forma

$$
\begin{aligned}
T(r, t) & =\int_{r^{\prime}=a}^{b} G\left(r, t, r^{\prime}, \tau\right)_{\tau=0} F\left(r^{\prime}\right) r^{\prime} d r^{\prime}+\frac{\kappa}{k} \int_{0}^{t} \int_{a}^{b} G\left(r, t, r^{\prime}, \tau\right) g\left(r^{\prime}, \tau\right) r^{\prime} d r^{\prime} d \tau+ \\
& +\kappa \int_{r^{\prime}=a}^{b}\left[r^{\prime} \frac{\partial G}{\partial r^{\prime}}\left(r, t, r^{\prime}, \tau\right)\right]_{r^{\prime}=a} f_{1}(\tau) d \tau-\kappa \int_{a}^{b}\left[r^{\prime} \frac{\partial G}{\partial r^{\prime}}\left(r, t, r^{\prime}, \tau\right)\right]_{r^{\prime}=b} f_{2}(\tau) d \tau
\end{aligned}
$$

O problema desta solução geral é que os dois últimos termos integrais que carregam informações de temperaturas prescritas nas fronteiras podem não convergir uniformemente em $r=a$ ou em $r=b$, para os valores de $f_{1}(t)$ ou $f_{2}(t)$, ou seja, condições de fronteira não homogêneas podem levar a dificuldades de convergência da solução próxima a elas (ÖZISIK, 1993). Um exemplo dessa dificuldade de convergência da solução pode ser visto no problema 8.8, cuja solução é dada pelas equações (8) a (138), de Hahn e Özisik (2012, p. 331). Uma das alternativas para aliviar essa dificuldade, segundo os referidos autores, é fatiar o problema original usando a metodologia proposta por Özisik (1993), que será aqui adotada e descrita resumidamente. Tal metodologia transforma em alguns casos, em particular o que estamos analisando, problemas não homogêneos de condução de calor em regime transiente com condições de fronteira não homogêneas, em problemas não homogêneos, mas com condições de 
fronteira homogêneas. É claro que se as condições de fronteira não homogêneas forem transformadas por essa técnica em homogêneas, a solução dada pela equação (47) não deverá ter mais as contribuições dos termos integrais como funções de $f_{1}(\tau)$ e $f_{2}(\tau)$.

Será assumida que a solução do problema dado pelas equações (1) a (4) pode ser separada pela contribuição de três componentes na forma

$$
T(r, t)=\theta(r, t)+\varphi_{1}(r) f_{1}(t)+\varphi_{2}(r) f_{2}(t),
$$

onde $\varphi_{1}(r)$ e $\varphi_{2}(r)$ satisfazem os seguintes problemas de Cauchy dados pelas equações diferenciais ordinárias na forma

$$
\begin{aligned}
& r \frac{d^{2} \varphi_{1}(r)}{d r^{2}}+\frac{d \varphi_{1}(r)}{d r}=0, \quad a<r<b, \\
& \varphi_{1}(a)=1, \quad \varphi_{1}(b)=0
\end{aligned}
$$

e

$$
\begin{aligned}
& r \frac{d^{2} \varphi_{2}(r)}{d r^{2}}+\frac{d \varphi_{2}(r)}{d r}=0, \quad a<r<b, \\
& \varphi_{2}(a)=0, \quad \varphi_{2}(b)=1 .
\end{aligned}
$$

Fazendo $r \frac{d \varphi_{i}(r)}{d r}=B_{i}, \quad i=1,2$, obtém-se $\varphi_{i}(r)=A_{i}+B_{i} \ln (r), \quad i=1,2$. Daí, e usando as condições de fronteira dadas pelas equações (49) e (50), resulta que

$$
\varphi_{1}(r)=\frac{\ln \left(\frac{r}{b}\right)}{\ln \left(\frac{a}{b}\right)} \text { e } \varphi_{2}(r)=\frac{\ln \left(\frac{r}{a}\right)}{\ln \left(\frac{b}{a}\right)} .
$$

As funções $\varphi_{i}(r)$ são as soluções estacionárias dos problemas de condução de calor em cilindro oco 1-D sem geração de calor, dado pelas equações (49) e (50). Das equações (1) a (4), (48) e (51), resulta o problema em $\theta=\theta(r, t)$ dado por

$$
\begin{aligned}
& \frac{\partial^{2} \theta}{\partial r^{2}}+\frac{1}{r} \theta+g^{*}=\frac{1}{k} \frac{\partial \theta}{\partial t}, \quad a<r<b, \quad t>0 \\
& C F 1: \quad \theta(a, t)=0, \quad t>0, \\
& C F 2: \quad \theta(b, t)=0, \quad t>0, \\
& \text { C.I : } \quad \theta(r, 0)=F^{*}(r), \quad a \leq r \leq b,
\end{aligned}
$$

onde

$$
g^{*}(r, t)=\frac{g(r, t)}{k}-\frac{1}{\kappa}\left[\varphi_{1}(r) \frac{d}{d t} f_{1}(t)+\varphi_{2}(r) \frac{d}{d t} f_{2}(t)\right]
$$

e 


$$
F^{*}(r)=F(r)-\left[\varphi_{1}(r) f_{1}(0)+\varphi_{2}(r) f_{2}(0)\right] .
$$

As equações (53) e (54) correspondem às equações (1.50a) e (1.50b) de Özisik (1993, p. 23). Esse método pode ser estendido a problemas multidimensionais, desde que as condições de fronteira sejam somente função do tempo.

A função de Green dada pela equação (41) pode ser usada para a solução do problema dado pela equação (52) na forma

$$
\theta(r, t)=\int_{a}^{b} G\left(r, t, r^{\prime}, \tau\right)_{\tau=0} F^{*}\left(r^{\prime}\right) r^{\prime} d r^{\prime}+\kappa \int_{0}^{t}\left(\int_{a}^{b} G\left(r, t, r^{\prime}, \tau\right) g^{*}\left(r^{\prime}, \tau\right) r^{\prime} d r^{\prime}\right) d \tau .
$$

Das equações (48) e (55), resulta a solução aproximada por funções de Green para o problema dado pelas equações (1) a (4) como

$$
\begin{aligned}
T(r, t)= & \int_{a}^{b} G\left(r, t, r^{\prime}, \tau\right)_{\tau=0} F^{*}\left(r^{\prime}\right) r^{\prime} d r^{\prime}+\kappa \int_{0}^{t}\left(\int_{a}^{b} G\left(r, t, r^{\prime}, \tau\right) g^{*}\left(r^{\prime}, \tau\right) r^{\prime} d r^{\prime}\right) d \tau+ \\
& +\varphi_{1}(r) f_{1}(t)+\varphi_{2}(r) f_{2}(t),
\end{aligned}
$$

onde $G\left(r, t, r^{\prime}, \tau\right)$ é dado como na equação (41) e $\varphi_{i}(r), i=1,2$, são dadas como na equação (51). Note que agora a solução obtida pela equação (56) converge para os valores prescritos na fronteira pelas equações (2) e (3), isto é,

$$
T(a, t)=f_{1}(t) \text { e } T(b, t)=f_{2}(t), \quad t>0 .
$$

Para ver isso, basta usar as equações (28) e (51) em $r=a$ e a equações (29) e (51) em $r=b$. Note que o primeiro termo do lado direito da equação (55) é a contribuição da condição inicial $F^{*}(r)$ de $\theta(r, t)$ para $T(r, t)$, isto é, a função de Green é evoluída em $\tau=0$, multiplicada por $F^{*}(r)$ e depois integrada no domínio $a \leq r \leq b$. O segundo termo traz a contribuição do termo de geração $g^{*}\left(r^{\prime}, \tau\right)$ de $\theta(r, t)$ para $T(r, t)$, ou seja, é a função de Green multiplicada por $g^{*}\left(r^{\prime}, \tau\right)$ e depois integrada nesse espaço. O terceiro termo traz as contribuições das condições de fronteira de Dirichlet. Das equações (41), (51), (54), a equação (56) pode então ser reescrita da seguinte forma

$$
\begin{aligned}
T(r, t) & =\frac{\pi^{2}}{2} \sum_{n=1}^{\infty} \frac{\lambda_{n}^{2} J_{0}\left(\lambda_{n} a\right)}{J_{0}^{2}\left(\lambda_{n} a\right)-J_{0}^{2}\left(\lambda_{n} b\right)} e^{-\kappa \lambda_{n}^{2} t} G_{0}\left(\lambda_{n} r\right) \int_{a}^{b} F^{*}\left(r^{\prime}\right) r^{\prime} G_{0}\left(\lambda_{n} r^{\prime}\right) d r^{\prime}+ \\
& +\frac{\kappa \pi^{2}}{2} \sum_{n=1}^{\infty} \frac{\lambda_{n}^{2} J_{0}\left(\lambda_{n} a\right)}{J_{0}^{2}\left(\lambda_{n} a\right)-J_{0}^{2}\left(\lambda_{n} b\right)} e^{-\kappa \lambda_{n}^{2} t} G_{0}\left(\lambda_{n} r\right) \int_{0}^{b} \int_{a}^{b} e^{-\kappa \lambda_{n}^{2} \tau} G_{0}\left(\lambda_{n} r^{\prime}\right) g^{*}\left(r^{\prime}, \tau\right) r^{\prime} d r^{\prime} d \tau+ \\
& +\frac{\ln \left(\frac{r}{b}\right)}{\ln \left(\frac{a}{b}\right)} f_{1}(t)+\frac{\ln \left(\frac{r}{a}\right)}{\ln \left(\frac{b}{a}\right)} f_{2}(t),
\end{aligned}
$$

onde as equações (53) e (54) são novamente apresentadas na forma 


$$
\begin{aligned}
g^{*}(r, t) & =\frac{g(r, t)}{k}-\frac{1}{\kappa}\left[\varphi_{1}(r) \frac{d}{d t} f_{1}(t)+\varphi_{2}(r) \frac{d}{d t} f_{2}(t)\right], \\
F^{*}(r) & =F(r)-\left[\varphi_{1}(r) f_{1}(0)+\varphi_{2}(r) f_{2}(0)\right],
\end{aligned}
$$

enquanto os $\lambda_{n}$ são dados pela equação (29) como

$$
G_{0}(\lambda r)=\left[J_{0}(\lambda r) Y_{0}(\lambda b)-J_{0}(\lambda b) Y_{0}(\lambda r)\right]=0 .
$$

Alguns casos especiais da equação (58) serão objetos de análise usando essa equação geral para uma aproximação da solução de problemas dados pelas equações (1) a (4).

A Figura 1 mostra um longo e fino cilindro oco de alumínio homogêneo e isotrópico, $L: a \leq r \leq b$, com condições de Dirichlet dependentes somente do tempo e com geração de calor.

Figura 1 - Cilindro oco de alumínio, longo e fino com condições de Dirichlet dependentes do

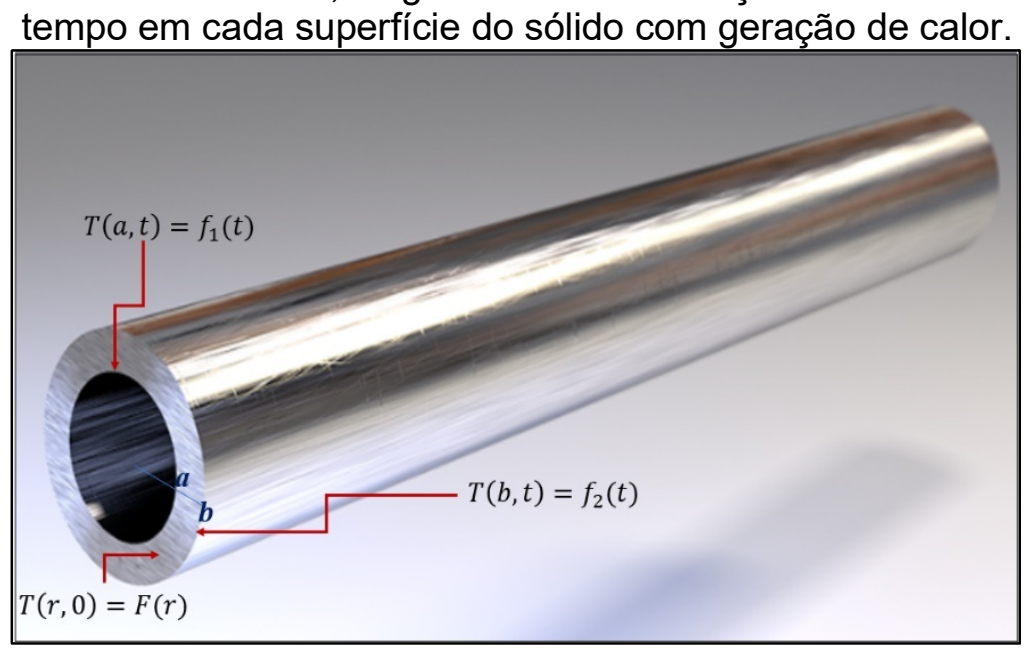

Fonte: Elaboração dos autores (2020).

Vamos considerar, de modo aproximado, os seguintes parâmetros para o alumínio: $k=190\left(\mathrm{~W} / \mathrm{m}^{\circ} \mathrm{K}\right), \quad a=0.045 \mathrm{~mm}, \quad b=0.075 \mathrm{~mm} \quad$ e $\kappa=80 \times 10^{-6}\left(\mathrm{~m}^{2} / \mathrm{s}\right)$. As unidades de temperatura e tempo no Sistema Internacional de Unidades (SI) foram convertidas para graus e minutos, respectivamente.

\subsection{Caso I.A}

Vamos considerar o cilindro da Figura 1, sujeito às seguintes condições de Dirichlet, sem geração de calor e condição inicial constante,

$$
\begin{aligned}
& T(a, t)=T(b, t)=0, \\
& g(r, t) \equiv 0, \\
& F(r)=V .
\end{aligned}
$$

Das equações (59) e (60), tem-se

$$
F^{*}(r)=F(r)=V \quad e \quad g^{*} \equiv 0 .
$$

Das equações (58), (60) e (61), resulta a solução desse problema na forma 


$$
T(r, t)=\frac{V \pi^{2}}{2} \sum_{n=1}^{\infty} \frac{\lambda_{n}^{2} J_{0}\left(\lambda_{n} a\right)}{J_{0}^{2}\left(\lambda_{n} a\right)-J_{0}^{2}\left(\lambda_{n} b\right)} e^{-\kappa \lambda_{n}^{2} t} G_{0}\left(\lambda_{n} r\right) \int_{r^{\prime}=a}^{b} r^{\prime} G_{0}\left(\lambda_{n} r^{\prime}\right) d r^{\prime} .
$$

Das equações (46) e (62), tem-se

$$
T(r, t)=V \pi \sum_{n=1}^{\infty} e^{-\kappa \lambda_{n}^{2} t} \frac{J_{0}\left(\lambda_{n} a\right)}{J_{0}^{2}\left(\lambda_{n} a\right)-J_{0}^{2}\left(\lambda_{n} b\right)} G_{0}\left(\lambda_{n} r\right) .
$$

A equação (63) concorda com a equação (13) obtida por Carslaw e Jaeger (2011, p. 207) para esse caso.

\subsection{Caso I.B}

O cilindro da Figura 1 está sob as mesmas condições do Caso I.A, mas agora com geração de calor uniforme, ou seja,

$$
\begin{aligned}
& T(r=a, t)=T(r=b, t)=0, \\
& g(r, t) \equiv g_{0}, \\
& F(r)=V,
\end{aligned}
$$

onde $g_{0}$ é agora tomado como constante. Das equações (59) e (64), tem-se

$$
F *(r)=F(r)=V \quad e \quad g^{*} \equiv \frac{g_{0}}{k} .
$$

Das equações (58), (64) e (65), resulta a solução desse problema na forma

$$
\begin{aligned}
T(r, t) & =V \pi \sum_{n=1}^{\infty} e^{-\kappa \lambda_{n}^{2} t} \frac{J_{0}\left(\lambda_{n} a\right)}{J_{0}^{2}\left(\lambda_{n} a\right)-J_{0}^{2}\left(\lambda_{n} b\right)} G_{0}\left(\lambda_{n} r\right)+ \\
& +\frac{\pi^{2}}{2} \frac{\kappa}{k} g_{0} \sum_{n=1}^{\infty} e^{-\kappa \lambda_{n}^{2} t} \frac{\lambda_{n}^{2} J_{0}\left(\lambda_{n} a\right)}{J_{0}^{2}\left(\lambda_{n} a\right)-J_{0}^{2}\left(\lambda_{n} b\right)} G_{0}\left(\lambda_{n} r\right) \int_{\tau=0}^{t} \int_{r^{\prime}=a}^{b} e^{-\kappa \lambda_{n}^{2} \tau} G_{0}\left(\lambda_{n} r^{\prime}\right) r^{\prime} d r^{\prime} d \tau .
\end{aligned}
$$

Das equações (46) e (66), obtém-se a solução aproximada desse problema na forma

$$
\begin{aligned}
T(r, t) & =V \pi \sum_{n=1}^{\infty} e^{-\kappa \lambda_{n}^{2} t} \frac{J_{0}\left(\lambda_{n} a\right)}{J_{0}^{2}\left(\lambda_{n} a\right)-J_{0}^{2}\left(\lambda_{n} b\right)} G_{0}\left(\lambda_{n} r\right)+ \\
& +\frac{\pi g_{0}}{k} \sum_{n=1}^{\infty}\left\{\frac{J_{0}\left(\lambda_{n} a\right)}{\lambda_{n}^{2}\left[J_{0}\left(\lambda_{n} a\right)+J_{0}\left(\lambda_{n} b\right)\right]} G_{0}\left(\lambda_{n} r\right)-\frac{J_{0}\left(\lambda_{n} a\right)}{\lambda_{n}^{2}\left[J_{0}\left(\lambda_{n} a\right)+J_{0}\left(\lambda_{n} b\right)\right]} G_{0}\left(\lambda_{n} r\right) e^{-\kappa \lambda_{n}^{2} t}\right\}
\end{aligned}
$$

Observe que a equação (67) "carrega" a solução dada pela equação (63), que continha somente a contribuição da condição inicial. Quando $t \rightarrow \infty, T(r, t) \rightarrow T_{S S}(r)$, isto é, a temperatura tende para o regime estacionário $T_{S S}(r)$. A equação estacionária em um cilindro oco 1-D com geração de calor pode ser obtida da equação (15) de Carslaw e Jaeger (2011, p. 191) na forma $T_{S S}(r)=A+B \ln r-\frac{g_{0} r^{2}}{4 k}$. Daí, e usando as condições de fronteira desse problema, podemos 
identificar o somatório do primeiro termo entre chaves da equação (67) com a solução estacionária, isto é,

$$
\begin{aligned}
\frac{\pi g_{0}}{k} \sum_{n=1}^{\infty} \frac{J_{0}\left(\lambda_{n} a\right) G_{0}\left(\lambda_{n} r\right)}{\lambda_{n}^{2}\left[J_{0}\left(\lambda_{n} a\right)+J_{0}\left(\lambda_{n} b\right)\right]} & \equiv-\frac{g_{0}}{\ln \left(\frac{a}{b}\right)} \frac{\left(a^{2}-b^{2}\right)}{4 k} \ln (a)+\frac{g_{0} a^{2}}{4 k}+ \\
& +\frac{g_{0}}{\ln \left(\frac{a}{b}\right)} \frac{\left(a^{2}-b^{2}\right)}{4 k} \ln (r)-\frac{g_{0} r^{2}}{4 k}=T_{S S}(r) .
\end{aligned}
$$

Introduzindo a equação (68) na equação (67), obtém-se a solução desse problema dada por

$$
\begin{aligned}
T(r, t)= & V \pi \sum_{n=1}^{\infty} e^{-\kappa \lambda_{n}^{2} t} \frac{J_{0}\left(\lambda_{n} a\right)}{J_{0}\left(\lambda_{n} a\right)+J_{0}\left(\lambda_{n} b\right)} G_{0}\left(\lambda_{n} r\right)-\frac{\pi g_{0}}{k} \sum_{n=1}^{\infty} e^{-\kappa \lambda_{n}^{2} t} \frac{J_{0}\left(\lambda_{n} a\right)}{\lambda_{n}^{2}\left[J_{0}\left(\lambda_{n} a\right)+J_{0}\left(\lambda_{n} b\right)\right]} G_{0}\left(\lambda_{n} r\right)+ \\
& -\frac{g_{0}}{\ln \left(\frac{a}{b}\right)} \frac{\left(a^{2}-b^{2}\right)}{4 k} \ln (a)+\frac{g_{0} a^{2}}{4 k}+\frac{g_{0}}{\ln \left(\frac{a}{b}\right)} \frac{\left(a^{2}-b^{2}\right)}{4 k} \ln (r)-\frac{g_{0} r^{2}}{4 k} .
\end{aligned}
$$

Em particular, se adicionalmente $V=0$, a equação (69) fica na forma

$$
\begin{aligned}
T(r, t) & =-\frac{\pi g_{0}}{k} \sum_{n=1}^{\infty} e^{-\kappa \lambda_{n}^{2} t} \frac{J_{0}\left(\lambda_{n} a\right)}{\lambda_{n}^{2}\left[J_{0}\left(\lambda_{n} a\right)+J_{0}\left(\lambda_{n} b\right)\right]} G_{0}\left(\lambda_{n} r\right)-\frac{g_{0}}{\ln \left(\frac{a}{b}\right)} \frac{\left(a^{2}-b^{2}\right)}{4 k} \ln (a)+ \\
& +\frac{g_{0} a^{2}}{4 k}+\frac{g_{0}}{\ln \left(\frac{a}{b}\right)} \frac{\left(a^{2}-b^{2}\right)}{4 k} \ln (r)-\frac{g_{0} r^{2}}{4 k} .
\end{aligned}
$$

A Figura 2 mostra uma simulação com os casos I.A e I.B, usando, respectivamente, as equações (63) e (70).

Figura 2 - Perfis de temperaturas no cilindro 1-D: (a) sem geração de calor e (b) com geração de calor.
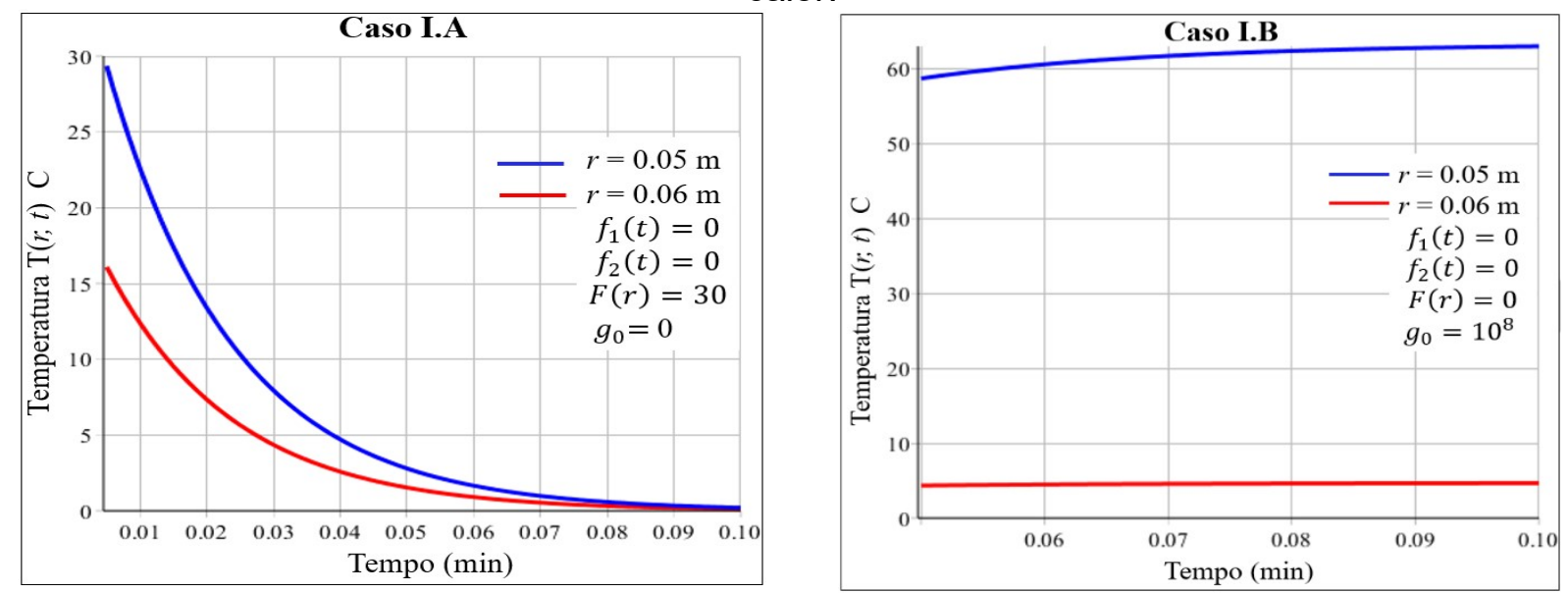

Fonte: Elaboração dos autores (2020). 
No caso I.A, pode ser notado o decaimento da temperatura a zero mais acentuado próximo à região em $r=a$, que está sob o efeito da condição de temperatura nula nessa superfície. No caso I.B, nota-se somente o efeito do termo de geração de calor no perfil de temperaturas. Observe que os gráficos foram gerados perto da fronteira, em $r=a$ e com tempos pequenos.

\subsection{Caso II.A}

O cilindro da Figura 1 está sujeito às seguintes condições de Dirichlet constantes e não necessariamente nulas, sem geração de calor, e condição inicial como função da variável espacial, isto é,

$$
\begin{aligned}
& T(a, t)=V_{1}, \quad T(b, t)=V_{2}, \\
& g(r, t) \equiv 0, \\
& T(r, 0)=F(r) .
\end{aligned}
$$

Das equações (59) e (71), tem-se

$$
F *(r)=F(r)-\left[\frac{V_{1} \ln \frac{b}{r}+V_{2} \ln \frac{r}{a}}{\ln \frac{b}{a}}\right]
$$

e

$$
g *(r, t) \equiv 0 .
$$

Aqui, a solução estacionária para esse problema de condução de calor é dada por

$$
T_{S S}(r)=\frac{V_{1} \ln \frac{b}{r}+V_{2} \ln \frac{r}{a}}{\ln \frac{b}{a}} .
$$

Das equações (58), (72) e (73), tem-se

$$
\begin{aligned}
T(r, t)= & \frac{\pi^{2}}{2} \sum_{n=1}^{\infty} \lambda_{n}^{2} \frac{J_{0}^{2}\left(\lambda_{n} a\right)}{J_{0}^{2}\left(\lambda_{n} a\right)-J_{0}^{2}\left(\lambda_{n} b\right)} e^{-\kappa \lambda_{n}^{2} t} G_{0}\left(\lambda_{n} r\right) \int_{a}^{b} G_{0}\left(\lambda_{n} r^{\prime}\right) F\left(r^{\prime}\right) r^{\prime} d r^{\prime}+\frac{V_{1} \ln \frac{b}{r}+V_{2} \ln \frac{r}{a}}{\ln \frac{b}{a}}+ \\
& -\frac{\pi^{2}}{2} \sum_{n=1}^{\infty} \lambda_{n}^{2} \frac{J_{0}^{2}\left(\lambda_{n} a\right)}{J_{0}^{2}\left(\lambda_{n} a\right)-J_{0}^{2}\left(\lambda_{n} b\right)} e^{-\kappa \lambda_{n}^{2} t} G_{0}\left(\lambda_{n} r\right) \int_{a}^{b} G_{0}\left(\lambda_{n} r^{\prime}\right)\left\{\frac{V_{1} \ln \frac{b}{r}+V_{2} \ln \frac{r}{a}}{\ln \frac{b}{a}}\right\} r^{\prime} d r^{\prime} .
\end{aligned}
$$

Usando a integral reportada por Carslaw e Jaeger (2011, p. 207), pode-se obter

$$
\int_{a}^{b} G_{0}\left(\lambda_{n} r^{\prime}\right)\left(\frac{V_{1} \ln \frac{b}{r}+V_{2} \ln \frac{r}{a}}{\ln \frac{b}{a}}\right) r^{\prime} d r^{\prime}=\frac{2\left(-V_{1} J_{0}\left(\lambda_{n} b\right)+V_{2} J_{0}\left(\lambda_{n} a\right)\right)}{\pi \lambda_{n}^{2} J_{0}\left(\lambda_{n} a\right)} .
$$

Das equações (75) e (76), resulta a expressão dada por 


$$
\begin{aligned}
T(r, t) & =\frac{\pi^{2}}{2} \sum_{n=1}^{\infty} \lambda_{n}^{2} \frac{J_{0}^{2}\left(\lambda_{n} a\right)}{J_{0}^{2}\left(\lambda_{n} a\right)-J_{0}^{2}\left(\lambda_{n} b\right)} e^{-\kappa \lambda_{n}^{2} t} G_{0}\left(\lambda_{n} r\right) \int_{a}^{b} G_{0}\left(\lambda_{n} r^{\prime}\right) F\left(r^{\prime}\right) r^{\prime} d r^{\prime}+ \\
& -\pi \sum_{n=1}^{\infty} \frac{\left[-V_{1} J_{0}\left(\lambda_{n} b\right)+V_{2} J_{0}\left(\lambda_{n} a\right)\right]}{J_{0}^{2}\left(\lambda_{n} a\right)-J_{0}^{2}\left(\lambda_{n} b\right)} J_{0}\left(\lambda_{n} a\right) e^{-\kappa \lambda_{n}^{2} t} G_{0}\left(\lambda_{n} r\right)+\frac{V_{1} \ln \frac{b}{r}+V_{2} \ln \frac{r}{a}}{\ln \frac{b}{a}} .
\end{aligned}
$$

A equação (77) concorda com a equação (15) de Carslaw e Jaeger (2011, p. 207) para esse caso; os autores usaram o método de separação de variáveis por meio de uma translação da solução estacionária. Em particular, se na equação (71), $V_{1}=V_{2}$ e $F(r)=V_{0}$, usando a equação (46), a equação (77) pode ser colocada na forma

$$
T(r, t)=\pi V_{0} \sum_{n=1}^{\infty} \frac{J_{0}\left(\lambda_{n} a\right)}{J_{0}\left(\lambda_{n} a\right)+J_{0}\left(\lambda_{n} b\right)} G_{0}\left(\lambda_{n} r\right) e^{-\kappa \lambda_{n}^{2} t}-\pi V_{1} \sum_{n=1}^{\infty} \frac{J_{0}\left(\lambda_{n} a\right)}{J_{0}\left(\lambda_{n} a\right)+J_{0}\left(\lambda_{n} b\right)} G_{0}\left(\lambda_{n} r\right) e^{-\kappa \lambda_{n}^{2} t}+V_{1} .
$$

Essa equação pode ser facilmente adaptada para aplicação em difusão de substâncias (CRANK, 2011) substituindo $\kappa$ por $D$ e $V_{i}$ pela concentração de substância $C_{i}$.

\subsection{Caso II.B}

O cilindro da Figura 1 está sujeito às seguintes condições de Dirichlet constantes e não necessariamente nulas, com geração de calor uniforme, $g_{0}$, e condição inicial como função da variável espacial, isto é,

$$
\begin{aligned}
& T(a, t)=V_{1}, \quad T(b, t)=V_{2}, \\
& g(r, t) \equiv g_{0}, \\
& T(r, 0)=F(r) .
\end{aligned}
$$

Das equações (59) e (79), tem-se

$$
g *(r, t)=\frac{g_{0}}{k} .
$$

Basta introduzir na equação (77) o segundo termo que aparece no lado direito da equação (58), isto é,

$$
\begin{aligned}
& T(r, t)=\frac{\pi^{2}}{2} \sum_{n=1}^{\infty} \lambda_{n}^{2} \frac{J_{0}^{2}\left(\lambda_{n} a\right)}{J_{0}^{2}\left(\lambda_{n} a\right)-J_{0}^{2}\left(\lambda_{n} b\right)} e^{-\kappa \lambda_{n}^{2} t} G_{0}\left(\lambda_{n} r\right) \int_{a}^{b} G_{0}\left(\lambda_{n} r^{\prime}\right) F\left(r^{\prime}\right) r^{\prime} d r^{\prime}+ \\
& -\pi \sum_{n=1}^{\infty} \frac{\left\{-V_{1} J_{0}\left(\lambda_{n} b\right)+V_{2} J_{0}\left(\lambda_{n} a\right)\right\}}{J_{0}^{2}\left(\lambda_{n} a\right)-J_{0}^{2}\left(\lambda_{n} b\right)} J_{0}\left(\lambda_{n} a\right) e^{-\kappa \lambda_{n}^{2} t} G_{0}\left(\lambda_{n} r\right)+\frac{V_{1} \ln \frac{b}{r}+V_{2} \ln \frac{r}{a}}{\ln \frac{b}{a}}+ \\
& +\frac{\kappa \pi^{2}}{2} \sum_{n=1}^{\infty} \frac{\lambda_{n}^{2} J_{0}\left(\lambda_{n} a\right)}{J_{0}^{2}\left(\lambda_{n} a\right)-J_{0}^{2}\left(\lambda_{n} b\right)} e^{-\kappa \lambda_{n}^{2} t} G_{0}\left(\lambda_{n} r\right) \int_{0}^{t} \int_{a}^{b} e^{-\kappa \lambda_{n}^{2} \tau} G_{0}\left(\lambda_{n} r^{\prime}\right) g^{*}\left(r^{\prime}, \tau\right) r^{\prime} d r^{\prime} d \tau .
\end{aligned}
$$


A integral dupla que aparece na equação (81) pode ser resolvida separadamente na variável $\tau$, e depois na variável espacial $r$, usando a equação (46), obtendo-se

$$
\begin{gathered}
T(r, t)=\frac{\pi^{2}}{2} \sum_{n=1}^{\infty} \lambda_{n}^{2} \frac{J_{0}^{2}\left(\lambda_{n} a\right)}{J_{0}^{2}\left(\lambda_{n} a\right)-J_{0}^{2}\left(\lambda_{n} b\right)} e^{-\kappa \lambda_{n}^{2} t} G_{0}\left(\lambda_{n} r\right) \int_{a}^{b} G_{0}\left(\lambda_{n} r^{\prime}\right) F\left(r^{\prime}\right) r^{\prime} d r^{\prime}+ \\
-\pi \sum_{n=1}^{\infty} \frac{\left\{-V_{1} J_{0}\left(\lambda_{n} b\right)+V_{2} J_{0}\left(\lambda_{n} a\right)\right\}}{J_{0}^{2}\left(\lambda_{n} a\right)-J_{0}^{2}\left(\lambda_{n} b\right)} J_{0}\left(\lambda_{n} a\right) e^{-\kappa \lambda_{n}^{2} t} G_{0}\left(\lambda_{n} r\right)+\frac{V_{1} \ln \frac{b}{r}+V_{2} \ln \frac{r}{a}}{\ln \frac{b}{a}}+ \\
\left(-\frac{g_{0} \pi}{k} \sum_{n=1}^{\infty} \frac{J_{0}\left(\lambda_{n} a\right) G_{0}\left(\lambda_{n} r\right)}{\lambda_{n}^{2}\left[J_{0}\left(\lambda_{n} a\right)-J_{0}\left(\lambda_{n} b\right)\right]} e^{-\kappa \lambda_{n}^{2} t}+\frac{g_{0} \pi}{k} \sum_{n=1}^{\infty} \frac{J_{0}\left(\lambda_{n} a\right) G_{0}\left(\lambda_{n} r\right)}{\lambda_{n}^{2}\left[J_{0}\left(\lambda_{n} a\right)-J_{0}\left(\lambda_{n} b\right)\right]}\right) .
\end{gathered}
$$

Quando $t \rightarrow \infty, T(r, t) \rightarrow T_{S S}(r)$, isto é, das equações (68) e (82), a temperatura tende para

$$
\begin{aligned}
& \frac{\pi g_{0}}{k} \sum_{n=1}^{\infty} \frac{J_{0}\left(\lambda_{n} a\right) G_{0}\left(\lambda_{n} r\right)}{\lambda_{n}^{2}\left[J_{0}\left(\lambda_{n} a\right)+J_{0}\left(\lambda_{n} b\right)\right]}+\frac{V_{1} \ln \frac{b}{r}+V_{2} \ln \frac{r}{a}}{\ln \left(\frac{a}{b}\right)} \equiv-\frac{g_{0}}{\ln \left(\frac{a}{b}\right)} \frac{\left(a^{2}-b^{2}\right)}{4 k} \ln (a)+\frac{g_{0} a^{2}}{4 k}+ \\
& +\frac{g_{0}}{\ln \left(\frac{a}{b}\right)} \frac{\left(a^{2}-b^{2}\right)}{4 k} \ln (r)-\frac{g_{0} r^{2}}{4 k}+\frac{V_{1} \ln \frac{b}{r}+V_{2} \ln \frac{r}{a}}{\ln \left(\frac{a}{b}\right)}=T_{S S}(r) .
\end{aligned}
$$

A equação (83) pode, ainda, ser colocada como

$$
T_{S S}(r)=V_{1}+g_{0} \frac{\left(a^{2}-r^{2}\right)}{4 k}+\frac{1}{\ln \left(\frac{a}{b}\right)}\left\{V_{1}-V_{2}+g_{0} \frac{\left(a^{2}-r^{2}\right)}{4 k}\right\} \ln \left(\frac{r}{a}\right) \text {. }
$$

É fácil verificar que $T_{S S}(a)=V_{1}$ e $T_{S S}(b)=V_{2}$. Das equações (82) e (84), tem-se

$$
\begin{aligned}
T(r, t) & =\frac{\pi^{2}}{2} \sum_{n=1}^{\infty} \lambda_{n}^{2} \frac{J_{0}^{2}\left(\lambda_{n} a\right)}{J_{0}^{2}\left(\lambda_{n} a\right)-J_{0}^{2}\left(\lambda_{n} b\right)} e^{-\kappa \lambda_{n}^{2} t} G_{0}\left(\lambda_{n} r\right) \int_{a}^{b} G_{0}\left(\lambda_{n} r^{\prime}\right) F\left(r^{\prime}\right) r^{\prime} d r^{\prime}+ \\
& -\pi \sum_{n=1}^{\infty} \frac{-V_{1} J_{0}\left(\lambda_{n} b\right)+V_{2} J_{0}\left(\lambda_{n} a\right)}{J_{0}^{2}\left(\lambda_{n} a\right)-J_{0}^{2}\left(\lambda_{n} b\right)} J_{0}\left(\lambda_{n} a\right) e^{-\kappa \lambda_{n}^{2} t} G_{0}\left(\lambda_{n} r\right)+V_{1}+g_{0} \frac{\left(a^{2}-r^{2}\right)}{4 k} . \\
& +\frac{1}{\ln \frac{a}{b}}\left\{V_{1}-V_{2}+g_{0} \frac{\left(a^{2}-r^{2}\right)}{4 k}\right\} \ln \frac{r}{a}-\frac{g_{0} \pi}{k} \sum_{n=1}^{\infty} \frac{J_{0}\left(\lambda_{n} a\right) G_{0}\left(\lambda_{n} r\right)}{\lambda_{n}^{2}\left[J_{0}\left(\lambda_{n} a\right)-J_{0}\left(\lambda_{n} b\right)\right]} e^{-\kappa \lambda_{n}^{2} t} .
\end{aligned}
$$

A equações (82) e (85) podem ser usadas para aproximar a solução do problema dado pelo caso II.B.

A Figura 3 mostra uma simulação com os casos II.A e II.B usando, respectivamente, as equações (77) e (85). 
Figura 3 - Perfis de temperatura no cilindro 1-D: (a) sem geração de calor e (b) com geração de calor.
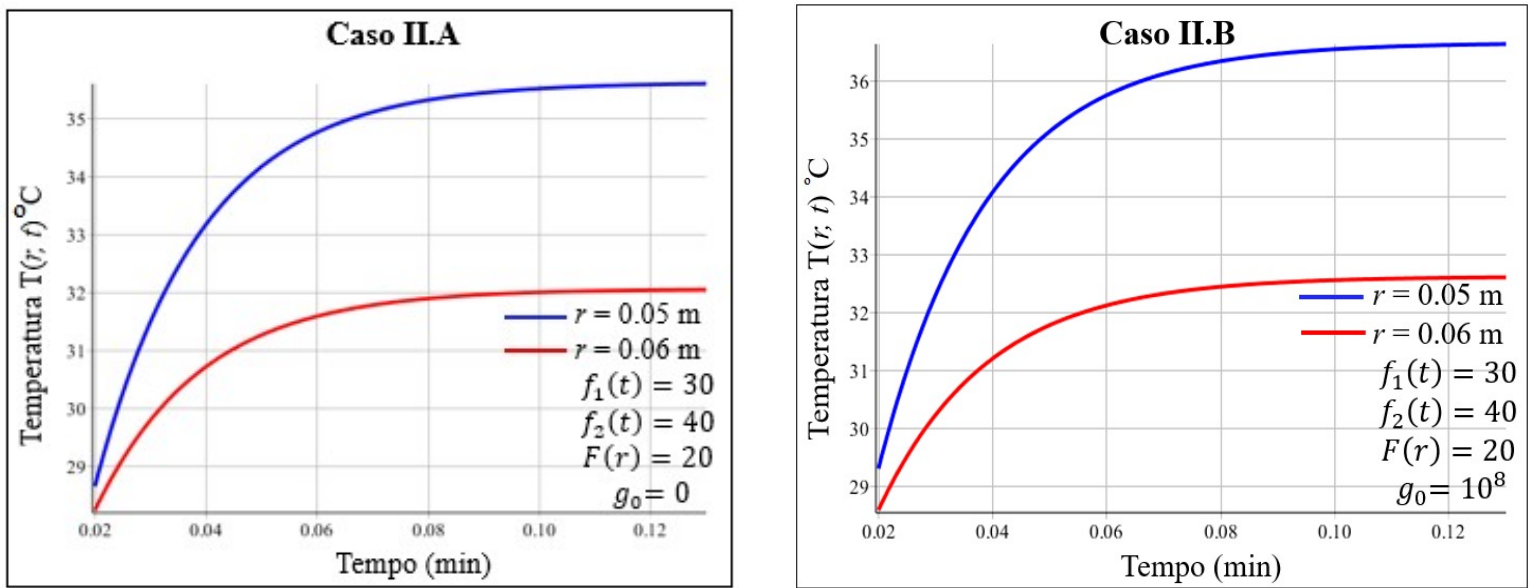

Fonte: Elaboração dos autores (2020).

Pode ser notado que a fonte de calor uniforme não produziu um aumento significativo de temperatura no cilindro, mostrando que as condições de Dirichlet de temperaturas constantes parecem limitar o aumento de temperatura produzido pela fonte de calor uniforme.

A Figura 4 mostra uma simulação desse problema, mas agora considerando um termo fonte variável. Para gerar esse gráfico foi usada a equação (81), pois a integração no tempo não fornece uma fórmula fechada como o ocorrido com a fonte uniforme de calor no caso anterior, dado pela equação (84).

Figura 4 - Perfis de temperatura no cilindro 1-D com geração de calor uniforme variável no tempo.

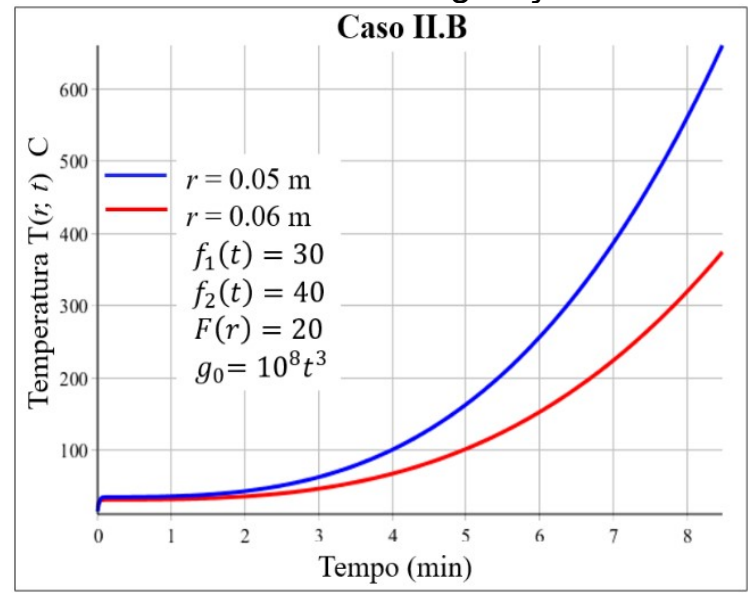

Fonte: Elaboração dos autores (2020).

Esses perfis de temperaturas mostram um aumento de temperatura rápida para a fusão do alumínio que ocorre em torno de $660{ }^{\circ} \mathrm{C}$. Pode ser notada uma maior variação de temperatura na posição $r=0.06 m$, em relação à região mais próxima da superfície interna em $r=a$. Isso pode ser devido à maior influência da condição de Dirichlet na superfície externa em $r=b$, que tem uma temperatura prescrita mais elevada. 
O último caso considera uma condição de Dirichlet variável no tempo na superfície em $r=b$.

\subsection{Caso III}

O cilindro da Figura 1 está sujeito às seguintes condições de Dirichlet, sendo uma constante e a outra variável, com geração de calor uniforme, $g_{0}$, e condição inicial de valor constante como função da variável espacial $r$, isto é,

$$
\begin{aligned}
& T(a, t)=V_{1}, \quad T(b, t)=\beta t, \\
& g(r, t) \equiv g_{0}, \\
& T(r, 0)=V_{0},
\end{aligned}
$$

onde $\beta$ é uma constante positiva. Das equações (59) e (86), tem-se

$$
g^{*}(r, t)=\frac{g_{0}}{k}-\frac{\beta}{\kappa}\left[\frac{\ln \left(\frac{r}{a}\right)}{\ln \left(\frac{b}{a}\right)}\right] \quad \text { e } \quad F^{*}(r, t)=V_{0}-V_{1}\left[\frac{\ln \left(\frac{b}{r}\right)}{\ln \left(\frac{b}{a}\right)}\right] .
$$

Das equações (58) e (87), resulta a expressão da solução aproximada desse problema, dada por

$$
\begin{aligned}
& T(r, t)=\frac{\pi^{2}}{2} \sum_{n=1}^{\infty} \frac{\lambda_{n}^{2} J_{0}^{2}\left(\lambda_{n} a\right)}{J_{0}^{2}\left(\lambda_{n} a\right)-J_{0}^{2}\left(\lambda_{n} b\right)} e^{-\kappa \lambda_{n}^{2} t} G_{0}\left(\lambda_{n} r\right) \int_{a}^{b} G_{0}\left(\lambda_{n} r^{\prime}\right)\left[V_{0}-V_{1} \frac{\ln \left(\frac{b}{r}\right)}{\ln \left(\frac{b}{a}\right)}\right] r^{\prime} d r^{\prime}+ \\
& +\frac{\pi^{2}}{2} \kappa \sum_{n=1}^{\infty} \frac{\lambda_{n}^{2} J_{0}^{2}\left(\lambda_{n} a\right)}{J_{0}^{2}\left(\lambda_{n} a\right)-J_{0}^{2}\left(\lambda_{n} b\right)} e^{-\kappa \lambda_{n}^{2} t} G_{0}\left(\lambda_{n} r\right) \int_{0}^{t} \int_{a}^{b} e^{\kappa \lambda_{n}^{2} \tau} G_{0}\left(\lambda_{n} r^{\prime}\right)\left[\frac{g_{0}}{k}-\frac{\beta}{\kappa} \frac{\ln \left(\frac{b}{r}\right)}{\ln \left(\frac{b}{a}\right)}\right] r^{\prime} d r^{\prime} d \tau . \\
& +V_{1} \frac{\ln \left(\frac{r}{b}\right)}{\ln \left(\frac{a}{b}\right)}+\beta\left[\frac{\ln \left(\frac{r}{a}\right)}{\ln \left(\frac{b}{a}\right)}\right] t
\end{aligned}
$$

A Figura 5 mostra perfis de temperaturas para o caso III.A quando $g_{0} \equiv 0$, comparado com o caso III.B com $g_{0}$ constante. Os gráficos foram gerados com a equação (88). A Figura 5(a) mostra a distribuição de temperaturas para os casos III.A e III.B em duas posições do cilindro, enquanto a Figura 5(b) mostra alguns perfis de temperaturas ao longo do domínio espacial com o tempo fixado. Nessa figura, pode ser notado que nas superfícies do cilindro oco, as temperaturas convergem para o mesmo valor das condições de Dirichlet, com ou sem calor. Esse fato era esperado e mostra a qualidade da solução obtida para esse problema com a equação (88). 
Figura 5 - Perfis de temperatura no cilindro 1-D

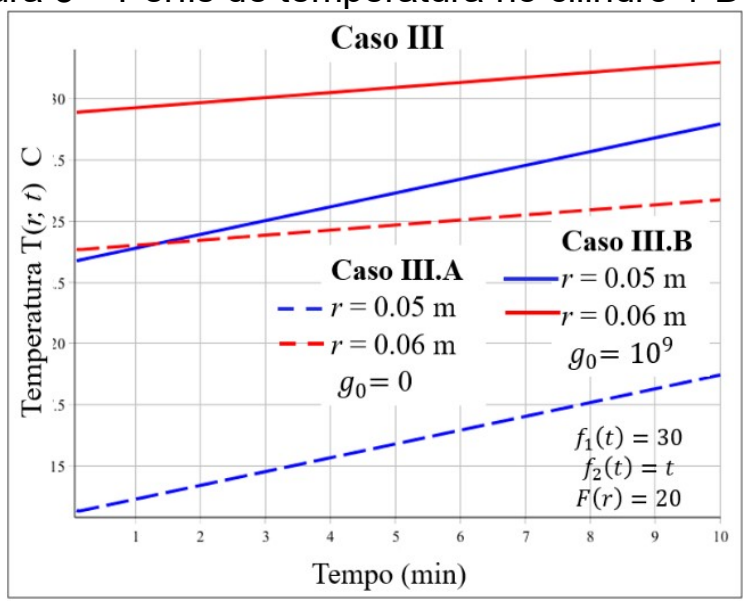

(a) sem geração calor e (b) com geração de calor.

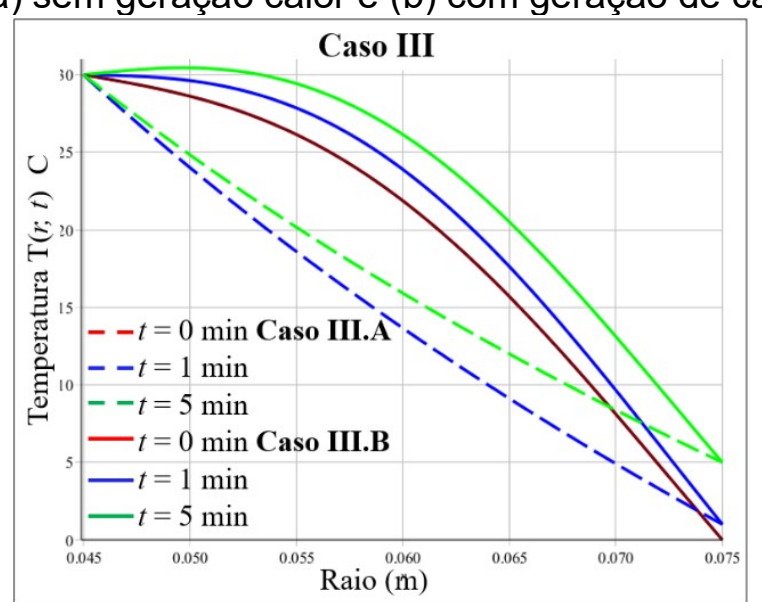

Fonte: Elaboração dos autores (2020).

\section{Conclusões}

Uma solução aproximadamente geral para o problema de condução de calor 1-D em regime transiente, com geração de calor em cilindros ocos homogêneos e isotrópicos, com condição de Dirichlet em função do tempo nas superfícies, e uma dada condição inicial foi aqui estabelecida. Para aliviar, ou atenuar possíveis dificuldades de convergência da solução nas proximidades das superfícies que constituem a região de distribuição de temperatura, foi adotado um método conhecido, que consiste em transformar as condições de fronteira não homogêneas em homogêneas, que combinadas com a função de Green obtida da solução aproximada da versão homogênea do problema analisado, tornou possível a obtenção de uma solução aproximadamente geral para esse tipo de problema. Simulações foram realizadas com essa equação, algumas delas envolvendo casos especiais reportados na literatura, o que permitiu a comparação dos resultados obtidos observando-se uma boa concordância entre eles. Desse modo, essa solução permite a análise de problemas térmicos mais gerais que possam ter aplicações na engenharia. Além disso, essa solução pode ser utilizada para a validação de soluções numéricas aproximadas aumentando a precisão delas. Uma importante integral formada por funções de Bessel integrante da expressão geral foi resolvida analiticamente, e o seu resultado utilizado na solução geral para obter expressões simplificadas dos casos especiais aqui analisados.

\section{Referências}

CARSLAW, H. S.; JAEGER, J. C. Conduction of heat in solids. 2. ed. Oxford: Clarendon Press, 2011.

CINELLI, G. An extension of the finite Hankel transform and applications. International Journal of Engineering Science, v. 3, n. 5, p. 539-559, out. 1965. DOI: https://doi.org/10.1016/00207225(65)90034-0.

CRANK, J. The mathematics of diffusion. 2. ed. Oxford: Clarendon Press, 2011. 
DAVIS, H. F. Fourier Series and Orthogonal Functions. New York: Dover Publications, Inc., 1963.

FERNANDES, A. P. Funções de Green: soluções analíticas aplicadas em problemas inversos de condução de calor. Orientador: Gilmar Guimarães. 2009. 130 f. Dissertação (Mestrado em Engenharia Mecânica) - Faculdade de Engenharia Mecânica, Universidade Federal de Uberlândia, Uberlândia, 2009. Disponível em: https://repositorio.ufu.br/bitstream/123456789/14844/1/ana.pdf. Acesso em: 28 jun. 2021.

FOX, R. W.; PRITCHARD, P. J.; MCDONALD, A. T. Introdução à mecânica dos fluidos.

Tradução e revisão técnica: Ricardo Koury e Luiz Machado. 7. ed. Rio de Janeiro: LTC, 2015.

HAHN, D. W.; ÖZISIK, M. N. Heat conduction. New Jersey: John Wiley \& Sons, 2012.

MEYER, R. E. Introduction to Mathematical Fluid Dynamics. New York: John Wiley \& Sons, 2007.

ÖZISIK, M. N. Heat conduction. New York: John Wiley \& Sons, 1993.

PERROTTA, J. A. Curso de introdução à engenharia do núcleo de reatores. São Paulo: Instituto de Pesquisas Energéticas e Nucleares, jul. 1999. Disponível em: https://www.ipen.br/portal por/conteudo/posgraduacao/arquivos/200906040956360-apperrotta.pdf. Acesso em: 5 jan. 2020.

RODRIGUES, R. R; MESQUITA, A. Z. Desenvolvimento de sistema para inspeção de elementos combustíveis de reatores nucleares de pesquisa Triga. Revista Tecnológica, Fortaleza, v. 38, n. 2, p. 1-13, 2017. DOI: https://doi.org/10.5020/23180730.2017.V38.2.7222.

ROMER, R. Você sabe o que são os cilindros que ficam no final dos cabos ligados ao seu PC? Canaltech, 8 mar. 2013. Disponível em: https://canaltech.com.br/produtos/Voce-sabe-o-que-saoos-cilindros-que-ficam-no-final-dos-cabos-ligados-ao-seu-PC/. Acesso em: 10 jun. 2020.

SOTOMAYOR, J. Lições de equações diferenciais ordinárias. Projeto Euclides. Rio de Janeiro: IMPA, 1979. 